Original Research Article

\title{
Evaluation of results of anterior cruciate ligament reconstruction using peroneus longus graft
}

\author{
Kautilyakumar V. Mahida*, Jyotish G. Patel, Hiren K. Shah, Ankit R. Patel
}

Department of Orthopaedics, GCS Medical College, Ahmedabad, Gujarat, India

Received: 20 February 2021

Accepted: 19 March 2021

\section{*Correspondence:}

Dr. Kautilyakumar V. Mahida,

E-mail: kautilya18496@gmail.com

Copyright: (C) the author(s), publisher and licensee Medip Academy. This is an open-access article distributed under the terms of the Creative Commons Attribution Non-Commercial License, which permits unrestricted non-commercial use, distribution, and reproduction in any medium, provided the original work is properly cited.

\section{ABSTRACT}

Background: The objective of the study was to assess clinical outcome and donor site morbidity of ACL reconstruction with peroneus longus tendon autografts in patients with ACL injury.

Methods: 60 Patients who underwent ACL reconstruction using peroneus longus autograft after fulfilling inclusion criteria and obtaining informed consent were assessed preoperatively and postoperatively and followed up for 1 year. Graft diameter was measured intraoperatively. Functional score of knee (Tegner and Lysholm Knee score) and American Orthopedic Foot and Ankle Score (AOFAS) for donor site morbidity were recorded preoperatively and 1 year after surgery.

Results: $93.3 \%$ Patients (56 out of 60) had good to excellent Lysholm knee score 1 year postoperatively and the mean AOFAS score was 96.7. The average peroneus longus graft diameter $8.7 \mathrm{~mm}$.

Conclusions: Anterior cruciate ligament reconstruction with peroneus longus autografts produces a good functional outcome at 1 year follow-up, with the advantages of large graft diameter and excellent ankle function based on AOFAS score.res.

Keywords: Anterior cruciate ligament reconstruction, Arthroscopy, Peroneus longus auto graft, Lysholm knee score, AOFAS score

\section{INTRODUCTION}

Anterior cruciate ligament (ACL) reconstruction improves knee stability and function with many graft types, either autografts or allografts, which have already been studied extensively. ${ }^{1,2}$ Among these grafts, bone-patellar tendonbone (BPTB) and four-strand hamstring autografts are the two most common autografts used for ACL reconstruction and each has its advantages and disadvantages. ${ }^{1}$ According to the newest studies, BPTB is the best graft choice because it has bone-to-bone healing which allows the successful incorporation of tunnel and graft, leading to a earlier return to function and sports activity. This aspect is important in professional athletes with ACL injuries. However, it carries the risk of patellar fracture, with an invasive approach and a large incision, fixed length and a weaker than native ACL, making it unsuitable for double- bundle reconstruction. ${ }^{3}$ Painfree kneeling is considered very important in the Asian population, especially in Indians, who kneel rigorously when praying which is not possible with BPTB graft. ${ }^{3}$ For these reasons, hamstring autografts are becoming popular in the Asian population. ${ }^{4}$

Peroneus longus tendon autografts are commonly used in some orthopaedic procedures, including spring ligament reconstruction, deltoid ligament reconstruction and medial patellofemoral ligament (MPFL) reconstruction (anterior half of the peroneus longus tendon). This is possible, due to the synergistic function of the peroneus longus and peroneus brevis. ${ }^{5}$ Some studies have even found that the peroneus brevis is a more effective evertor of the ankle, justifying the harvest of the peroneus longus tendon. ${ }^{5}$ 
Some previous case series reported using the peroneus longus tendon as the first choice for an autograft in ACL reconstruction, with good clinical outcome and minimal donor site morbidity while other studies did not agree, due to donor site morbidity. ${ }^{6,7}$ Phatama et al reported that there was no significant difference between the peroneus longus and hamstring tendon in terms of tensile strength. ${ }^{8}$

Hence there are very few studies and controversy exist regarding use and the results of ACL reconstruction using Peroneus longus autograft. The purpose of this study is to evaluate the functional outcomes of ACL reconstruction using Peroneus longus autograft using the Tegner and Lysholm knee score and also evaluate the morbidity at the donor site by using the American Orthopedic Foot and Ankle Score (AOFAS) at 6 months follow-up..$^{9,10}$

\section{METHODS}

This prospective study had been conducted in the Department of Orthopedics, GCS Medical College and Research Hospital, Ahmedabad. The diagnosis of ACL rupture was established and the patients gave their informed consent to be included in this study. Sixty patients underwent single-bundle ACL reconstruction from January 2019 to June 2020. The peroneus longus tendon was used as an autograft in the Arthroscopic ACL reconstruction in these patients.

The inclusion criteria were patients with an isolated rupture of the ACL, age 16-45 years. The exclusion criteria were as follows: associated ligament injury, chondral damage, meniscal injury, fracture around the knee and the presence of a pathological condition in the lower extremity or an abnormal contralateral knee joint. The functional score (Lysholm) was recorded before the surgery and 1 year after the surgery. The postoperative evaluation was performed 1 year after the surgery to give the patient enough time to complete the rehabilitation protocol and return to sports activity, as well as being likely to obtain peak function after an ACL injury.

\section{Operative technique}

The patients lay in a supine position under regional anesthesia and a tourniquet was applied to the thigh and inflated without elevation and exsanguination. Standard anterolateral and anteromedial portals were used. Diagnostic arthroscopy for ACL rupture and to rule out other ligament and meniscal injury was performed, followed by graft harvesting of the ipsilateral peroneus longus.

For the peroneus tendon, the location of the skin incision was marked, 2-3 cm above and $1 \mathrm{~cm}$ behind the lateral malleolus. The incision was made through the skin, subcutaneous tissue and superficial fascia. The peroneus longus and peroneus brevis tendons were identified. The location of the tendon division was marked, $2-3 \mathrm{~cm}$ above the level of the lateral malleolus. The distal part of the peroneus longus tendon to the peroneus brevis tendon was sutured with end-to-side sutures. The peroneus longus tendon was stripped proximally with a tendon stripper to about $4-5 \mathrm{~cm}$ from the fibular head to prevent peroneal nerve injury.

The intercondylar notch was then cleared of fibrous tissue to ease visualization during preparation of the tunnels. The femoral tunnel and the tibial tunnel were then prepared independently. After drilling the tunnels, we proceeded with the implantation of the tendon with graft fixation on the femoral side with a button and graft fixation on the tibial side with a bioabsorbable screw after appropriate tensioning.

\section{RESULTS}

During the study period, 60 patients met the inclusion criteria and underwent ACL reconstruction using peroneus longus graft. Intraoperatively, the diameter of the graft was measured and recorded and the result shows that the mean diameter of the peroneus longus graft was $8.7 \mathrm{~mm}$. Majority of the patients with an ACL injury reconstructed with peroneus longus tendon experienced a significant improvement in the Lysholm scores 1 year after the surgery, as shown in Table 1. About 93.3 percent of patients had good to excellent results in terms of lysholm knee score 1 year postoperative. Four patients $(6.6 \%)$ had fair result in terms of pain and mild instability of knee joint which was managed by oral analgesic, ice-application and physiotherapy. Other postoperative morbidity, stiffness, infection and re-rupture incidence were not noted in our study.

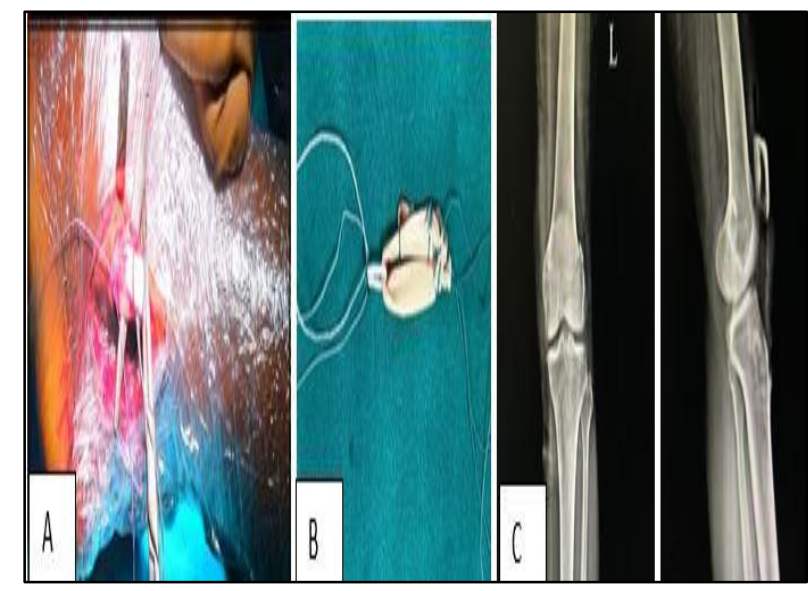

Figure 1: (A) Peroneus longus graft harvesting. (B) Graft preparation. (C) Postoperative radiographs from left to right.

\section{Donor site morbidity of peroneus longus autografts}

For the evaluation of donor site morbidity for peroneus longus tendon autografts, assessments of the functional score for the ankle using AOFAS scores were performed at 1 year follow up the mean AOFAS score for the donor ankle was 96.67 . 


\section{DISCUSSION}

Autograft choice is one of the most important considerations during ACL reconstruction surgery of the knee. We found that graft diameter peroneus longus tendons was greater when comparing to average graft diameter of hamstring tendons from other studies. ${ }^{1}$ The effect of the autograft diameter on the re-rupture and revision rate of the reconstructed ACL of the knee has been studied extensively. Some authors have shown that a reduction in autograft diameter is related to a higher revision rate, especially in younger patients. ${ }^{10}$

Previous studies reported good results after ACL reconstruction with the peroneus longus tendon, in terms of both functional outcome and knee stability. ${ }^{6}$ Our study has also shown comparable outcomes implying that the peroneus longus tendon can be used in ACL reconstruction with good and excellent functional outcomes for the patient at the 1 year follow up. While a longer term evaluation (at least 2 years) might be needed, it was shown in a previous study that functional outcome after ACL reconstruction showed minimal differences between the 1and 2-year follow-ups. ${ }^{12}$

A previous study by Angthong et al mentioned possible donor site morbidity using the peroneus longus tendon, including reduced peak torque eversion and inversion, decreased ankle function and concerns about ankle stability. ${ }^{7}$ In this study, we evaluated ankle function using the AOFAS score; the mean score was 96.67 which shows excellent function and is comparable to the results of study by Rhatomy et al. ${ }^{1}$ This is probably because the peroneus brevis is still intact in the donor ankle. Previous studies have mentioned that the peroneus brevis is a more effective evertor of the ankle, which will maintain the eversion function of the ankle after harvesting the peroneus longus tendon. ${ }^{5}$ Even though previous studies, like that by Angthong and Chernchujit, have mentioned a reduction in ankle peak torque eversion and inversion, we found that the functional outcome was still excellent. Further studies could focus on measurements of ankle stability and power and correlate them with functional scores after harvesting the peroneus longus tendon.

\section{CONCLUSION}

ACL reconstruction with peroneus longus tendon autografts had an excellent functional outcome (Lysholm score), with no donor site morbidity on evaluation.

Funding: No funding sources Conflict of interest: None declared

Ethical approval: The study was approved by the institutional ethics committee

\section{REFERENCES}

1. Rhatomy, Sholahuddin, Asikin , Asa, Wardani, Anggun et al. Peroneus longus autograft can be recommended as a superior graft to hamstring tendon in single-bundle ACL reconstruction. Knee Surgery, Sports Traumatology, Arthroscopy. 2019;27.

2. Frank CB, Jackson DW. The science of reconstruction of the anterior cruciate ligament. J Bone Joint Surg Am. 1997;79:1556-76.

3. Adam F, Pape D, Schiel K, Steimer O, Kohn D, Rupp S. Biomechanical properties of patellar and hamstring graft tibial fixation techniques in anterior cruciate ligament reconstruction. Am J Sports Med. 2004;32:71-8

4. Romanini E, D’Angelo F, De Masi S, Adriani E, Magaletti M, Lacorte E et al. Graft selection in arthroscopic anterior cruciate ligament reconstruction. J Orthop Traumatol. 2010;11:211-9.

5. Otis JC, Deland JT, Lee S, Gordon J. Peroneus brevis is a more effective evertor than peroneus longus. Foot Ankle Int. 2004;25:242-6.

6. Sasetyo DR, Rhatomy S, Pontoh LA. Peroneus longus tendon: the promising graft for anterior cruciate ligament reconstruction surgery. Asia Pac J Sport Med Arthrosc Rehabil Technol. 2017;9:25.

7. Angthong C, Chernchujit B, Apivatgaroon A, Chaijenkit K, Nualon P, Suchao-in K. The anterior cruciate ligament reconstruction with the peroneus longus tendon: a biomechanical and clinical evaluation of the donor ankle morbidity. J Med Assoc Thai. 2015;98:555-60.

8. Rudy ME, Phatama KY. Tensile strength comparison between peroneus longus and hamstring tendons: a biomechanical study. Int J Surg Open. 2017;9:41-4.

9. Tegner Y, Lysholm J, Odensten M, Gillquist J. Evaluation of cruciate ligament injuries A review Acta Orthopaedica Scandinavica. 1988;59(3):33641.

10. Esther M M, Van Lieshout A, Siebe De Boer, Duncan E, Meuffels P, Hoed TD et al. American Orthopaedic Foot and Ankle Society(AOFAS) Ankle-Hinfoot Score: a study protocol for thr translation and validation of the Dutch language version BMJ Open. 2017;7(2):e012884.

11. Magnussen RA, Lawrence JTR, West RL, Toth AP, Taylor DC, Garrett WE. Graft size and patient age are predictors of early revision after anterior cruciate ligament reconstruction with hamstring autograft. Arthroscopy. 2012;28:526-31.

12. Heijne A, Werner S. A 2-year follow-up of rehabilitation after ACL reconstruction using patellar tendon or hamstring tendon grafts: a prospective randomised outcome study. Knee Surg Sports Traumatol Arthrosc. 2010;18:805-13.

Cite this article as: Mahida KV, Patel JG, Shah HK, Patel AR. Evaluation of results of anterior cruciate ligament reconstruction using peroneus longus graft. Int J Res Orthop 2021;7:594-6. 